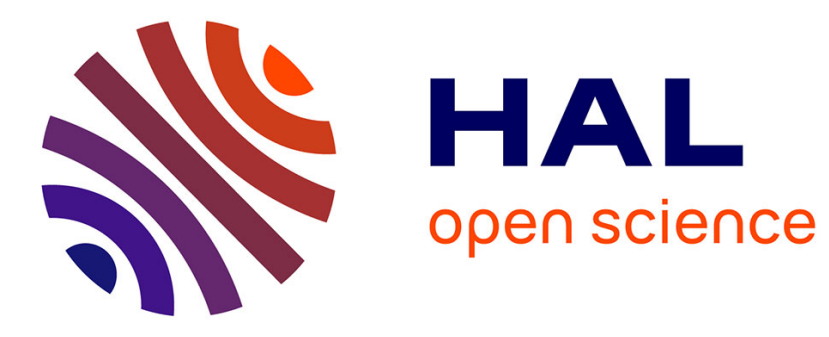

\title{
Modeling and robust control strategy for a control-optimized piezoelectric microgripper.
}

Mathieu Grossard, Mehdi Boukallel, Nicolas Chaillet, Christine

Rotinat-Libersa

\section{- To cite this version: \\ Mathieu Grossard, Mehdi Boukallel, Nicolas Chaillet, Christine Rotinat-Libersa. Modeling and robust control strategy for a control-optimized piezoelectric microgripper.. IEEE/ASME Transactions on Mechatronics, 2010, PP (99), pp.1-10. 10.1109/TMECH.2010.2050146 . hal-00504464}

\section{HAL Id: hal-00504464 \\ https://hal.science/hal-00504464}

Submitted on 20 Jul 2010

HAL is a multi-disciplinary open access archive for the deposit and dissemination of scientific research documents, whether they are published or not. The documents may come from teaching and research institutions in France or abroad, or from public or private research centers.
L'archive ouverte pluridisciplinaire HAL, est destinée au dépôt et à la diffusion de documents scientifiques de niveau recherche, publiés ou non, émanant des établissements d'enseignement et de recherche français ou étrangers, des laboratoires publics ou privés. 


\title{
Modeling and robust control strategy for a control-optimized piezoelectric microgripper
}

\author{
Mathieu Grossard, Mehdi Boukallel, Nicolas Chaillet, Member, IEEE, and Christine Rotinat-Libersa
}

\begin{abstract}
In this paper, modeling and robust control strategy for a new control-optimized piezoelectric microgripper are presented. The device to be controlled is a piezoelectric flexible mechanism dedicated to micromanipulation. It has been previously designed with an emphasis to control strategy, using a new topological optimization method, by considering innovative frequency-based criteria. A complete non-linear model relating the voltage and the resulting deflection is established taking into account hysteresis as a plurilinear model subjected to uncertainties. The approach used for controlling the actuator tip is based on a mixed High Authority Control (HAC) / Low Authority Control (LAC) strategy for designing a wide-band regulator. It consists of a Positive Position Feedback (PPF) damping controller approach combined with a low-frequency integral controller which is shown to have robustness performances as good as a RST-based robust pole placement approach for the microgripper. The rejection of the vibrations, naturally induced by the flexible structure, and the control of the tip displacement have been successfully performed. Because we had taken into account frequency-based criteria from the first designing step of our device, we demonstrate that the tuning of the HAC/LAC can be easily performed and leads to low regulator order.
\end{abstract}

Index Terms-Flexible structure, hysteresis, microrobotics, piezoelectric actuator, positive position feedback, robust control, vibrations control.

\section{INTRODUCTION}

$\mathbf{T}$ O achieve micromanipulation tasks, microgripper devices are often compliant mechanisms, i.e. singlebodies, elastic continua flexible structures that transmit a motion by undergoing elastic deformation [1]. They are opposed to jointed rigid body motions of conventional articulated mechanisms. These compliant mechanism-based micromanipulators are often coupled with smart materials for actuation, such as PZT (Lead Zirconate Titanate) piezoceramic. Piezoelectric actuation has become widespread in micromanipulation systems where high positioning accuracy is needed [2].

Manuscript received on May 5th, 2010.

M. Grossard and C. Rotinat-Libersa are with the Interactive Robotics Laboratory, LIST, CEA, F-92265 Fontenay aux Roses, France ;

M. Boukallel is with the Sensory and Ambient Interfaces Laboratory, LIST, CEA, F-92265 Fontenay aux Roses, France ;

$\mathrm{N}$. Chaillet is with the Automatic Control and Micro-Mechatronic Systems Department, FEMTO-ST Institute, Unité Mixte de Recherche CNRS 6174 -University of Franche-Comté (UFC)/Ecole Nationale Supérieure de Mécanique et des Microtechniques (ENSMM)/University of Technology of Belfort-Montbéliard (UTBM), F-25000 Besançon, France.

Corresponding author: Mathieu Grossard, CEA, LIST, Interactive Robotics Laboratory, 18 route du Panorama, BP6, FONTENAY AUX ROSES, F-92265

France ; E-mail: mathieu.grossard@cea.fr

phone: + 33146549 188, fax: + 33146548980

Paper type: regular paper.
Recently [3], we have developed a prototype of a new compliant piezoelectric monolithic gripper (Fig.1). It has been designed using a global systematic approach, based on the multidisciplinary topology optimization of the flexible structure. This method is based on the flexible building block method called FlexIn ("Flexible Innovation"), which uses a multidisciplinary genetic algorithm to optimize flexible structures [4] [5] [6]. Details on the design procedure can be found in [3], and are reminded in section II of this article. When the active beams of the symmetric jaws of the microgripper are supplied by voltage, it results in a deformed shape that produces a symmetric stroke of $\delta= \pm 10.51 \mu \mathrm{m}$ and a gripping force of about $0,84 \mathrm{~N}$ along $x$-axis under $\pm 100 \mathrm{~V}$. These static mechanical characteristics of our device are of the same order of magnitude of other well-known actuation schemes such as unimorph or bimorph PZT actuators, widespread in the design of microrobotic manipulators [1].

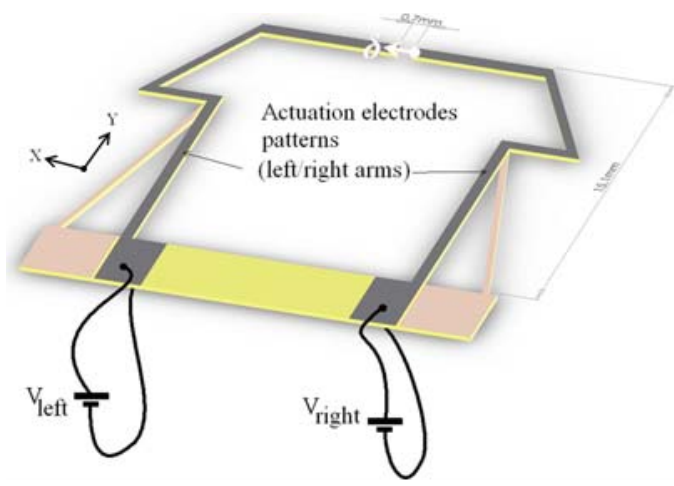

Fig. 1. 3D CAD model of the piezoeletric device with top face electrode patterns ( $V_{\text {left }}$ and $V_{\text {right }}$ are the controlled inputs for actuating the left and right arms).

However, when the amplitude of the applied voltage reaches high values (about $40 \mathrm{~V}$ ), the linear approximation between the deflection and the voltage is not valid anymore, and hysteresis phenomenon particularly arises [7]. It exists several methods to deal with such a nonlinearity : linearization methods (using charge compensation [8] for example), Preisach model [9] (but, due to its complexity, the real time implementation of this technique is difficult), linear or polynomial approximation models [10]. Let us note that an another cause of precision loss at the tip of the piezoelectric actuators is the drift due to creep effects [11]. Another major drawback in using flexible structure in micromanipulation tasks is the loss of position control accuracy due to vibrations. Methodologies used for synthesizing sophisticated robust controllers are not so intuitive and often lead to high order regulator. 
For the design of our microgripper (Fig.1), both purely mechanical criteria (i.e. stroke and force at the tip) and innovative frequency-based criteria [4] [3] have been used. These last criteria are useful tools to ensure the efficient control of flexible structures afterwards. These criteria allow to take into account resonance amplitude modulations and optimal pole/zero placement in the frequency spectrum of the device, so that the designer can fit its open-loop frequency response function in a desired way [12] [13] [14]. As a consequence, because our device has been frequency-optimized, it appears that the synthesis of a simple robust HAC/LAC regulator is easy afterwards. Indeed, this methodology, which combines damping controllers with lowfrequency integral controller, is particularly interesting for the control of flexible structure. It can guarantee interesting stability margins and lead to low order regulator compared with classic robust pole placement approaches.

This paper is organized as follows. In the next section, we briefly remind the underlying ideas of the control-oriented optimization strategy that lead to the specific design of our gripper (Fig.1). The non-linear voltage-deflection model including hysteresis is presented in section III. In section IV, the hysteresis model is identified and approximated by a quadrilateral linear model subjected to uncertainty and with a varying static gain. In the fourth part, a High Authority Control (HAC) / Low Authority Control (LAC) controller is synthesized and implemented to ensure the performances required in micromanipulation. Finally, in section VI, we show that our controller can have robustness as good as a RST-based robust pole placement approach for the microgripper.

\section{Presentation of the CONTROL-OPtimized COMPLIANT PIEZOACTUATOR PROTOTYPE SYNTHESIZED BY FLEXIN}

Reader can find detailed descriptions of the FlexIn optimization tool in [4] [5] [6]. The way the piezoelectrically actuated prototype has been optimally synthesized is extensively detailed in [3]. In this section, we point out the interests of having optimized our device from a control-oriented point of view, in order to ensure the performances required in closedloop afterwards.

From the first design step, we had the objective to conceive a flexible mechanism characterized by two dominant modes in the targeted low-frequency spectrum (reduced model) in order to facilitate the identification of an accurate reduced order model afterwards. In addition, the specific alternating pole/zero pattern for our flexible structure (i.e. the resonances and antiresonances alternate in this targeted spectrum) helps designing regulator, which has good intrinsic stability properties.

\section{A. Useful criteria for evaluation of dynamic input-output model performances of flexible systems}

Two significant tasks in flexible structure control are both the identification of the dominant modes to build an appropriate reduced model and the control strategy design.

1) Evaluation of the model reduction cost: Since the dynamic model of a flexible structure is characterized by a large number of resonant modes, accurate identification of all the dominant system dynamics often leads to high order models. A model reduction is often required.

Thus, to facilitate the computation of an accurate identified model afterwards, a first criterion has been drawn in FlexIn to optimize the reduced-model accuracy of the systems, while limiting spillover effects [3]. The optimal structures is the one guaranteeing the highest joint controllability and observability for all the modes in the bandwidth of interest, while providing the minimum joint controllability and observability of the neglected modes (Fig.2). The numerical formulation of this criterion can be found in [3].

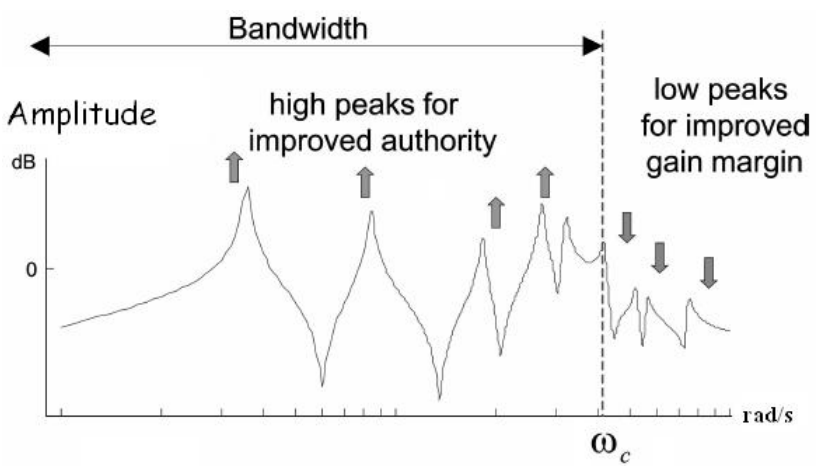

Fig. 2. Desired form of the open-loop magnitude FRF. Resonance peaks amplitudes must be maximized in the frequencies bandwidth $\left[0, \omega_{c}\right]$ to increase authority control on these dominant modes. On the contrary, the amplitudes of resonance peaks after cut-off frequency must be minimized to increase gain margin and to limit modes destabilization in this area (spillover phenomenon)

2) Pseudo-collocated behavior: For some specific class of flexible structures, which can be modeled as collocated resonant systems, active damping controllers such as Positive Position Feedback (PPF) have proven to offer great robustness, performance, and ease of implementation. They are often focused on damping the dominant modes [15], [12]. The most useful characteristic of a collocated system is the interlacing of poles and zeros along the imaginary axis for a lightly damped structure. Such systems are minimum of phase. This results in a phase response that lies continuously between $0^{\circ}$ and $180^{\circ}$. Minimum of phase systems are known to possess interesting properties, especially simple stability conditions. This makes collocation of the transfer function an attractive control approach.

Although the flexible structures rarely present natural collocated behavior, an other optimization criterion, inspired by [15] and [14], has been used to force the structure to have this collocated behavior in terms of frequency response function.

\section{B. Presentation of the monolithic compliant piezoactuator prototype}

From the optimal design obtained [3], a symmetric monolithic microactuation mechanism prototype has been fabri- 
cated, made of a single piezoelectric material PIC151 from PI Piezo Ceramic Technology [16]. Fig.1 shows the 3D CAD model of the device with top electrode patterns. The whole structure is divided into an active (electroded) and a passive areas, which both will be free to bend. The base remaining area will be clamped and kept out from bending (Fig.3). The clamping area is where the electric wires will feed the electrodes, respectively with $V_{\text {left }}$ and $V_{\text {right }}$ for the actuated left and right arms of the gripper.

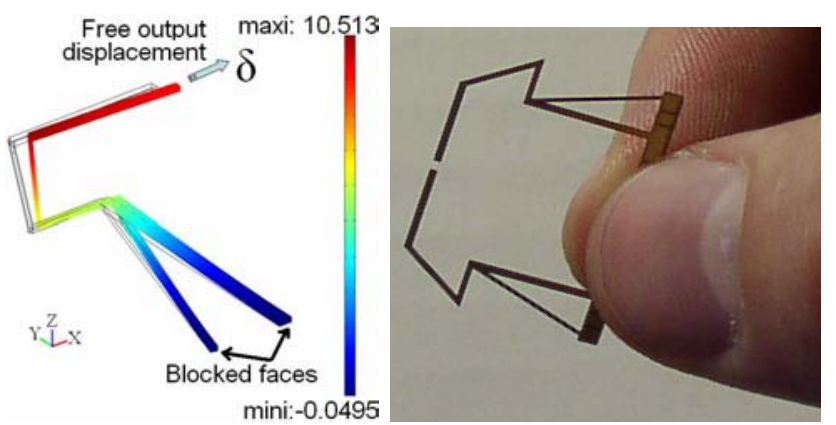

Fig. 3. On the left, $3 D$ simulation of the $x$-displacement $\delta$ (in $\mu m$ ) when the half piezoactuated structure is activated under $\pm 100 \mathrm{~V}$, and associated deformed shape. On the right, photo of the whole machined piezoelectric monolithic device.

\section{NON-LINEAR MODELING OF THE TRANSFER VOLTAGE-DEFLECTION}

In this section, we draw the electromechanical model of the piezoelectric device based on experimental measurements. It consists in a linear model subjected to both uncertainties and a time varying static gain. A complete model of the voltagedeflection transfer is considered as the series connection of a static hysteresis operator and linear dynamics [17] [18].

\section{A. Experimental setup}

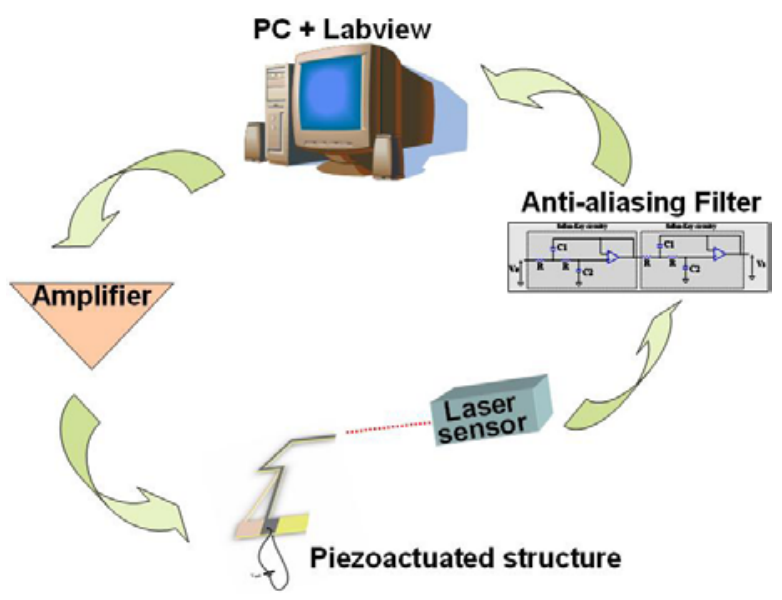

Fig. 4. Schematics of the experimental setup.

The microactuator prototype is clamped, and placed on $x$ $y$ - $z$ micropositioning linear stages, which are manually operated. The piezoelectric actuator requires high voltage (about $\pm 100 \mathrm{~V})$ to provide micrometric deflection. Thus, the device is connected to a linear power amplifier, with an amplification ratio $\times 50$. This last device is controlled via a computer equipped with Matlab-Simulink software and a NI Labview PXI board, whose sampling frequency is $f_{e}=20 k H z$ (Fig.4).

Output displacement at the tip of the piezoelectric structure is measured along $x$-axis using a $0.01 \mu \mathrm{m}$-resolution Keyence laser sensor. The analog output of the laser sensor is directly connected to a $4^{\text {th }}$-order low-pass anti-aliasing filter. In the following, we note $F(s)$ its transfer function, where $s$ is the Laplace variable. A double Sallen-Key circuitry is tuned for providing more than $75 \mathrm{~dB}$ attenuation at $f_{e} / 2=10 \mathrm{kHz}$ Shannon frequency (Fig.5) :

$$
F(s)=\frac{V_{s}(s)}{V_{e}(s)}=\left(\frac{1}{1+2 C_{2} R s+C_{1} C_{2} R^{2} s^{2}}\right)^{2}
$$

This filter eliminates data treatment errors that could result from aliasing and unmodeled high-frequency noise dynamics.

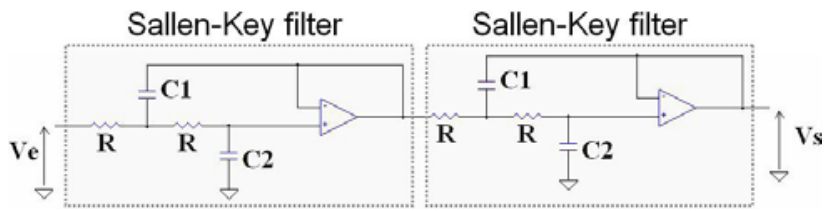

Fig. 5. The active anti-aliasing filter consists of two Sallen-Key circuitries in series. Chosen values of components are $R=2.2 \mathrm{k} \Omega, C_{1}=100 \mathrm{nF}$ and $C_{2}=47 n F$.

\section{B. Description of the piezoelectric actuator behavior}

General electromechanical relations adopted for the piezoelectrically actuated device are a function of the applied electrical $U$ and mechanical $F_{m}$ stimulations. $U$ refers to the voltage applied on the upper and lower electrodes of the microactuator, and $F_{m}$ to the mechanical force applied at the tip of the device. In the present article, our electromechanical model of the piezoelectric device is based on a parametric model, which has to be identified in experimentation. We choose to model the deflection $\delta$ along the $x$-axis using a control-oriented relationship that is currently adopted for the piezoelectric actuators [19]. For a more phenomenological point of view, a macroscopic thermodynamically constitutive law describing the hysteresis effects, which occur in ferroelectric ceramics such as PZT, can be found in [20] [21]. Let's note that a physical electromechanical model in finite element has been previously used for the design optimization of our device [3].

According to [19], the deflection is non-linearly linked to $U$ and $F_{m}$ as follows:

$$
\delta(s)=s_{p} D(s) F_{m}(s)+\Gamma(U(s), s)
$$

where $s_{p}$ is the elastic compliance, and $D(s)$ the dynamic part with $D(0)=1$. $\Gamma(U(s), s)$ is an operator that includes the hysteresis $H(U, s)$ and the creep $C(U, s)$ non-linearities in a decoupled way:

$$
\Gamma(U(s), s)=H(U, s)+C(U, s)
$$


Let note that notation of $\Gamma$ depends on both $U$ and $s$, since, in the general case, hysteresis depends on the past and present values of $U$ and also on its frequency.

The creep phenomenon $\delta_{\text {Creep }}$ is the drift of the deflection observed after the transient part, when a step voltage is applied to the piezoelectric actuator as shown in Fig.6. It can be considered as an additional behavior happening when the steady-state is reached, so that it is often modeled as a simple delayed transfer [22]. Experimental results show that the transient part of piezoelectric microactuators are generally less than $500 \mathrm{~ms}$ whereas the creep settling time is more than $180 \mathrm{~s}$ [23]. Usually, the creep is considered as a disturbance that the controlled system must reject.

Thus, in the following, $\Gamma(U, s)$ is assumed to be only modeled by $H(U, s)$ hysteresis term, which represents both the gain value and the transient part of the electromechanical transfer.

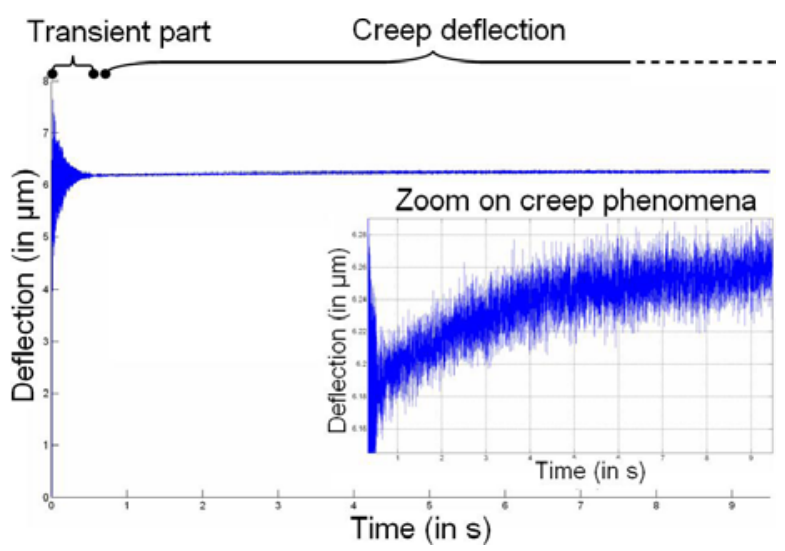

Fig. 6. Measured creep deflexion of the piezoelectric actuator when a $100 \mathrm{~V}$ voltage step is applied at $t=0 s$.

\section{Analysis of the hysteresis}

$\mathrm{A} \pm 50 \mathrm{~V}$ sine voltage input is applied to the active microactuator, and the displacement output at the tip of the device $\delta$ is recorded. No force is applied at the tip.

Experimental results on Fig.7 show the frequency-dependent behavior of the hysteresis phenomenon : the shape variation is due to linear vibrational dynamics [24].

According to [17], [25], [26], we propose to model this hysteresis by considering the decoupling of the hysteresis operator $H(U, s)$ into a static hysteresis part $H_{i}(U)$ of a constant shape in series with a linear dynamical part $D(s)$ (see Fig.8). (It has been proved that the transient part $D(s)$ is independent of the amplitude of the voltage [23].)

\section{IDENTIFICATION OF THE PIEZOACTUATOR MODEL}

In this section, we focus on the experimental identification of the piezoelectric actuator device. The vibrational dynamics are firstly identified. Then, the static hysteresis part is modeled and identified.
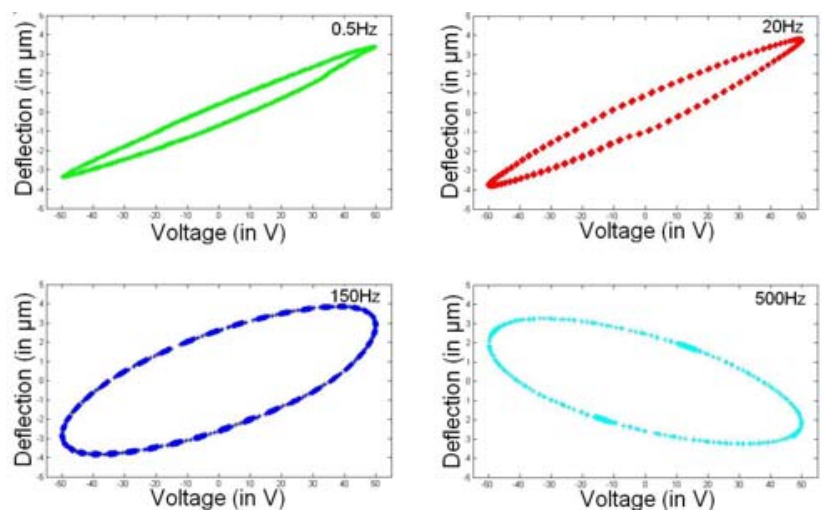

Fig. 7. Measured hysteresis of the piezoelectric actuator for various frequency voltage inputs.

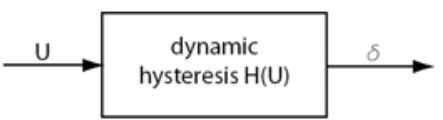

(a)

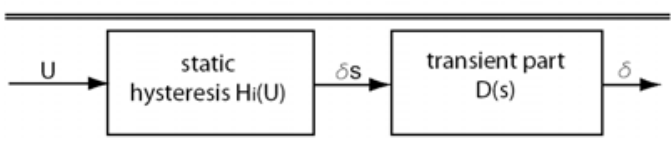

(b)

Fig. 8. Dynamic hysteresis equivalence [22].

\section{A. Identification of the vibrational dynamics}

To study and isolate the response due to the induced vibrations from the creep phenomenon, relatively highfrequency inputs were used. The input amplitudes were also kept small so that hysteresis effects could be negligible as well. The vibrational dynamics are recorded experimentally by applying to the piezoactuator a low-amplitude sine input $U$ of increasing frequency. Using a spectrum analyser device $(H P 3562 A)$, Bode diagram is recorded and $D(s)=\frac{\delta(s)}{U(s)}$ transfer is directly identified in Laplace domain.

As expected by FlexIn optimization, the two first resonances modes are dominant over the following vibrational modes, and the pole/zero alternate pattern is kept into this desired spectrum of interest (Fig.9). Identification process is thus performed considering these first vibrations modes involved in the reduced model. For identification, we consider a secondorder modal transfer expansion :

$$
D(s)=\sum_{i=1}^{2} \frac{k_{i}}{1+\frac{2 \xi_{i}}{\omega_{n i}} s+\frac{1}{\omega_{n i}^{2}} s^{2}}=\frac{N(s)}{M(s)}
$$

Damping ratio $\xi_{1}$ and $\xi_{2}$ are calculated from the measured quality factor at $-3 \mathrm{~dB}$ on the Bode diagram, as $Q_{i}^{-3 d B} \approx$ $\frac{1}{2 \xi_{i}}$. Then, values of the natural pulsation $\omega_{n 1}$ and $\omega_{n 2}$ are easily calculated thanks to the measured resonance frequencies (Fig.9). Identified modal damping and natural frequency values are mentioned in Table.I.

Finally, values of the static gain $k_{1}$ and $k_{2}$ are calculated to have both the right frequency value for the first antiresonance 
TABLE I

IDENTIFIED MODAL PARAMETERS OF $D$ TRANSFER FUNCTION.

\begin{tabular}{cc|cc}
\hline \hline$\xi_{1}$ & $\omega_{n 1}($ rad.s & \\
\hline $1.97 \%$ & 1597.1 & $2.12 \%$ & 5934.1 \\
\hline \hline
\end{tabular}

(which occurs between the two resonances) and the right static gain of the whole system, leading to :

$$
\begin{aligned}
N(s)= & 2.254 \times 10^{-8} \mathrm{~s}^{2}+1.466 \times 10^{-6} \mathrm{~s}+1 \\
M(s)= & 1.113 \times 10^{-14} \mathrm{~s}^{4}+3.499 \times 10^{-12} \mathrm{~s}^{3} \\
& +4.206 \times 10^{-7} \mathrm{~s}^{2}+3.178 \times 10^{-5} \mathrm{~s}+1
\end{aligned}
$$

Identified response model $D(s) F(s)$ is compared with the experimental frequency response taking into account the filter transfer $F(s)$ (Fig.9).
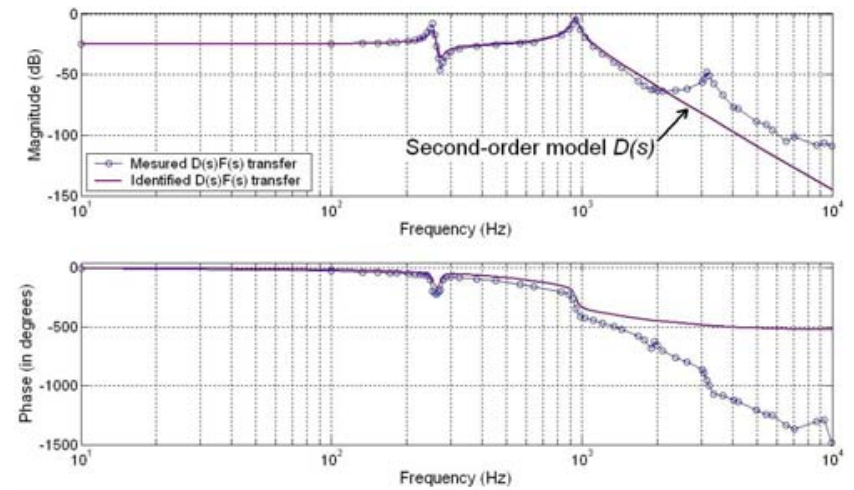

Fig. 9. Experimental Bode diagram of the $\frac{\delta}{U} \operatorname{transfer}(\delta$ in $\mu m$ and $U$ in Volt) and corresponding identified transfer $D(s) F(s)$ with scaled static gain to allow comparison.

\section{B. Validation of the hysteresis modeling}

In this paragraph, we demonstrate the following assumption $H(U, s)=H_{i}(U) \cdot D(s)$. This model relies on Fig.8, where $H_{i}(U)$ is a static hysteresis that represents the gain.

We compare the simulated model of $H_{i}(U) \cdot D(s)$ with the experimental curve of $H(U, s)$. The simulated model of $D(s)$ is given by the identified model in (4). Thus, a precise model of the static hysteresis $H_{i}(U)$ is needed.

The hysteresis model given by Coleman and al. in [27] has been chosen. This model has already been successfully applied to piezoelectric actuators in [28]. This model relies on the following differential equation :

$$
\left(H_{i}\right): \quad \dot{\delta}=\alpha|\dot{U}|(a U-\delta)+b \dot{U}
$$

The parameters $a=0.9998 \mu \mathrm{m} . V^{-1}, b=0.0635 \mu \mathrm{m} . V^{-1}$ and $\alpha=0.0140 V^{-1}$ are identified from the experimental hysteresis curve. For the identification of these parameters, the considered hysteresis curve is defined as the quasi-static stationary loop in $(0, U, \delta)$ plan. A $0,5 \mathrm{~Hz}$ sine voltage is chosen as an exciting signal. Indeed, such a frequency is quite low to neglect vibrational dynamics, but sufficiently high towards the cutoff frequency of the creep deflection $f_{c}^{\text {creep }} \approx \frac{1}{2 \pi(180)} \approx 0,9 \mathrm{mHz}$.

The comparison results between the experimental data and the simulated model show clearly that the hysteresis $H(U, s)$ is equivalent to a static hysteresis followed by a dynamic part: $H(U, s)=H_{i}(U) \cdot D(s)$ (see (Fig.10)).
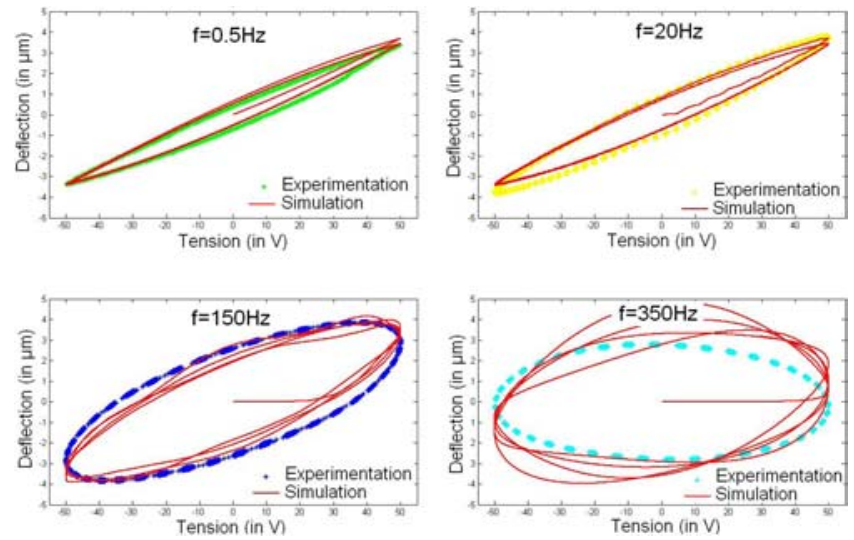

Fig. 10. Comparison of $H(U, s)$ (experimental result) and $H_{i}(U) \cdot D(s)$ (simulation) at different frequencies.

C. Presentation and identification of the plurilinear modeling for the static hysteresis $H_{i}(U)$

Although less accurate than non-linear models (such as Bouc-Wen or Preisach ones), the quadrilateral approximation method has been chosen for modeling the static hysteresis part, as proposed in [22]. This method presents the advantage of a simple model, involving very few numbers of parameters, which makes it very suitable for controller design purposes. The resulting uncertainties, which can be easily predicted, are supposed to be taken into account by the synthesis of a robust controller at the end.

The non-linear hysteresis curve is approximated by a piecewise affine function. In case of non-saturation, the hysteresis can reasonably be approximated by four straightlines (Fig.8). The static hysteresis $H_{i}(U)$ is replaced by a linear model with a nominal slope $\alpha_{0}$ and an offset $\delta_{H}$ :

$$
H_{i}(U)=\alpha_{0} U+\delta_{H}
$$

where $\alpha_{0}$ is defined by the middle value of the maximal $\alpha_{M}$ and the minimal $\alpha_{m}$ straightlines slopes values

$$
\alpha_{0}=\frac{1}{2}\left(\alpha_{M}+\alpha_{m}\right)
$$

and $\alpha_{E}$ by the slopes radius as follows:

$$
\alpha_{E}=\frac{1}{2}\left(\alpha_{M}-\alpha_{m}\right)
$$

Thus, the real static gain $\alpha_{\text {system }}$ of the system is characterized by

$$
\left\{\begin{array}{c}
H_{i}(U)=\alpha_{\text {system }} U+\delta_{H} \\
\alpha_{0}-\alpha_{E} \leq \alpha_{\text {system }} \leq \alpha_{0}+\alpha_{E}
\end{array}\right.
$$

It indicates that the nominal model (7) has uncertainty relative to the nominal static gain $\alpha_{0}$, determined by the radius $\alpha_{E}$. 
This approach consider $\delta_{H}$ as an additional disturbance Fig.11.

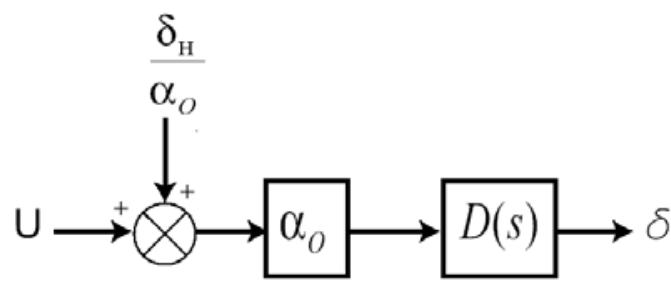

Fig. 11. Open-loop voltage-deflection model with quadrilateral hysteresis approximation.

For parameters estimation, the considered hysteresis loop is defined as the stationary loop in the input/output plane for a quasi-static oscillating input. A $0.5 \mathrm{~Hz}$ low-frequency sinusoid is chosen here: such a frequency is sufficiently small to avoid the dynamic effects, but important enough compared to the creep cut-off frequency $f_{c}^{\text {creep }} \approx \frac{1}{2 \pi 180} \approx 0.9 \mathrm{mHz}$. The chosen sine voltage amplitude is $100 \mathrm{~V}$, which is about the command range required by the controller afterwards. After computing the slope of each four straightlines fitting the $(U, \delta)$-plot, the values of $\alpha_{M}$ and $\alpha_{m}$ are deduced (see Fig.12), so that we obtain the nominal gain $\alpha_{0}=0.0640 \mu \mathrm{m} . V^{-1}$ and the uncertainty radius $\alpha_{E}=0.0122 \mu m . V^{-1}$ values.

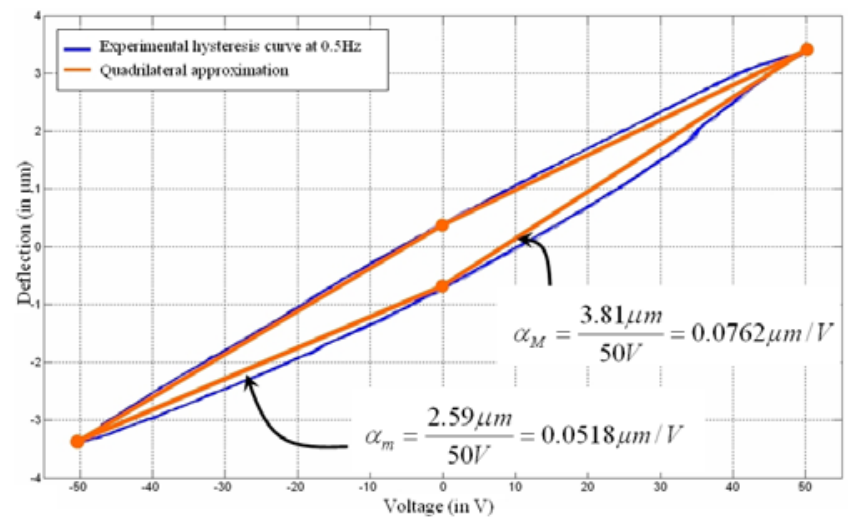

Fig. 12. Quadrilateral approximation of the hysteresis and identification of the parameters. (The segments ends are chosen at the ventral nodes of the hysteresis where amplitude of the deflection is maximal.)

\section{Complete dynamic model for controller synthesis}

The mechanical term $s_{p} D(s) F_{m}(s)$ in equation (2) describes the dynamic of the contact force with the manipulated object at the tip of the microactuator. As a consequence, $\delta_{F}$ deflection induced by force loads $F_{m}, \delta_{F}(s)=s_{p} D(s) F_{m}(s)$, is considered as a disturbance on the nominal deflection $\delta$.

So, the complete nominal dynamic model of the deflection, including the contribution of the anti-aliasing filter $F(s)$, takes the following form

$$
\delta(s)=F(s)\left(\alpha_{0} D(s) U(s)+\delta_{\text {Pert. }}(s)\right)
$$

where $\alpha_{0}$ is the nominal static gain completed by the radius uncertainty $\alpha_{E}$ to limit the real varying static gain of the system, such as $\alpha_{0}-\alpha_{E} \leq \alpha_{\text {system }}(t) \leq \alpha_{0}+\alpha_{E}$. The whole system dynamics is defined as $G(s)=\alpha_{0} D(s) F(s)$ in the following. The $\delta_{\text {Pert }}$. term gathers together the different perturbation sources (hysteresis offset, force loads and creep phenomenon), as follows:

$$
\delta_{\text {Pert. }}(s)=\delta_{H} D(s)+\delta_{F}(s)+\delta_{\text {Creep }}(s)
$$

Then, this model is used to synthesize our HAC/LAC controller.

\section{A HAC/LAC STRATEGY CONTROL FOR IN-PLANE MOTION CONTROL}

This approach consists of combining two different loops as shown on Fig.13:

- The active damping regulator (LAC) $H_{l}$ reduces the settling time of transient disturbances and the effect of steady state disturbances near the resonances frequencies of the system $G(s)$.

- A compensator $H_{L}$ design (HAC) achieves integral action at low frequency and significant modifications to the open-loop system poles dealing with the trade-off between the conflicting requirements of performancesrobustness-stability. This outer loop of the compensator is designed on the actively damped structure.

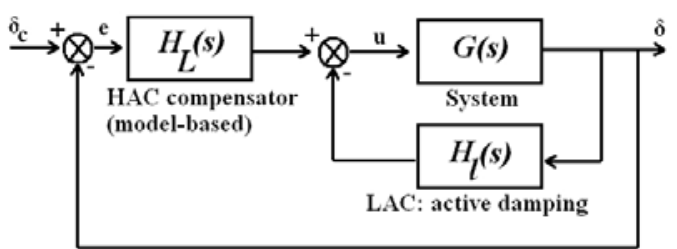

Fig. 13. Principle of the dual loop HAC/LAC control

\section{A. LAC regulator synthesis}

One way of adding damping to the structure is to use Positive Position Feedback (PPF), as proposed in [29]. This technique has the advantage to be stable even in the presence of uncontrolled modes in the bandwidth, and rolls off quickly at higher frequencies, reducing the risk of destabilizing systems with high-frequency dynamics.

Stable close-loop performances can be achieved by positively feeding back the position signals of the tip deflection to the compensator input. In SISO case, PPF transfer function is given by:

$$
H_{l}(s)=-g \frac{1}{1+\frac{2 \xi_{f}}{\omega_{f}} s+\frac{1}{\omega_{f}^{2}} s^{2}}
$$

where $g, \omega_{f}$ and $\xi_{f}$ are respectively the chosen gain, natural frequency and damping of controller mode. In our study, interests are brought on the damping of the first mode $\omega_{n 1}$. The second mode amplitude will be consequently attenuated by the $-40 \mathrm{~dB} /$ decade roll-off of the resulting transfer pre-compensated by this one-mode PPF controller. The experimental results that we get at the end demonstrate 
that damping our system with only one PPF is sufficient with respect to our specifications.

This second-order low-pass filter controller is tuned here to resonate at the first structural natural frequency. PPF control create auxiliary degrees-of-freedom (DOFs), i.e. controller modes, that produce particular modal movements of the system [30]. As a consequence, it adds a $\pi / 2 \mathrm{rad}$ phase at this frequency, so that the measured position and the applied control voltage are $\pi$ rad out of phase. Energy is dissipated from the $\omega_{n 1}$ mode in which the additional DOFs participate, thus adding damping at this frequency.

The tuning of the controller, represented by the three parameters $g>0, \omega_{f}$ and $\xi_{f}$, is done manually in an iterative way based on the root-locus plot Fig.14. Parameters values are chosen according to considerations based on each of these parameters influence.

1) Tuning of the PPF controller damping ratio: Larger values of the damping ratio $\xi_{f}$ will result in a less important slope in phase response at resonant frequency, thereby increasing the region of active damping. However, it is expected to result in a less effective damping, i.e. in an increased flexibility at lower modes. Here, after several trials in simulatio, we take $\xi_{f}=50 \%$ as a compromise.

2) Tuning of the PPF controller natural frequency: Influence of the parameter $\omega_{f}$ is rather straightforward since it makes the damping work either in a lower or higher frequency region. $\xi_{f}$ being imposed, damping the first natural mode $\omega_{n 1}$ implies to take $\omega_{f}$ equal to $2258 \mathrm{rad} / \mathrm{s}$ value. Thus, it puts the compensator resonant pole on the target natural frequency $\omega_{n 1}$ of the $G(s)$ system.

3) Tuning of the PPF controller gain: The influence of the gain variable is depicted in the root-loci plots (Fig.15).

Since the close-loop poles start at the undamped open-loop poles and end at the undamped zeros, it exists an optimal gain $g$ between them that ensures a maximal modal damping. As optimized for our microactuator, its frequency response presents interlacing pole/zero pattern until the second resonance. Thus, root-locus plot depict damping loop that remain entirely in the left half plane, guarantying robust stability [12], [29] (see Fig.15a for the first mode of $D(s)$ ).

Moreover, static gain $g$ ensures the close-loop system stability [29]. The interlacing property of the poles and zeros no longer holds after the second resonance occurrence, because the anti-aliasing filter $F(s)$ adds double poles after the second resonant mode $\omega_{n 2}$ of the flexible prototype (double pole at $\left.\omega_{\text {filter }} \approx 6280 \mathrm{rad} / \mathrm{s}\right)$. Thus, an unstable loop occurs when considering the second mode of $D(s)$ (see Fig.15b).

As a compromise between an optimal damping of the first mode (corresponding to a high value of $g$ ) and no-degraded stability performance for the second mode (poor value of $g$ ), we have chosen $g=4.7$ in the following Fig.14. Let's note that the real static gain $\alpha_{\text {system }}$ of the system is characterized by

$$
\alpha_{0}-\alpha_{E} \leq \alpha_{\text {system }} \leq \alpha_{0}+\alpha_{E}
$$

Thus, we have checked that the system compensated by such a choice of gain $g$ for the PPF controller is always stable for a static gain comprised between these lower and upper boundaries.

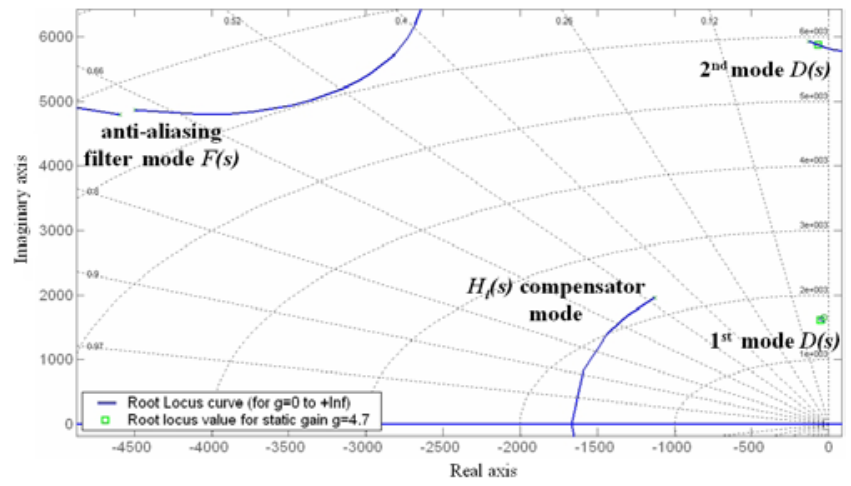

Fig. 14. Root locus of the close-loop (trajectories of the close-loop poles as a function of gain $g$ ) and static gain chosen. Graph is symmetric towards Real axis.
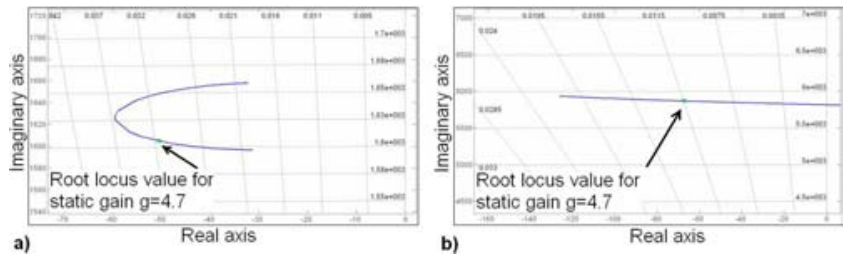

Fig. 15. Root locus zoomed on $D(s)$ first mode (a) and $D(s)$ second mode (b). Graph is symmetric towards Real axis.

4) Results: Such tuned parameters enable to increase the modal damping $\xi_{1}$ up to $3.14 \%$ in close-loop, instead of $1.97 \%$ in open-loop. Let's note that, although the LAC regulator tends to degrade the second modal damping $\left(\xi_{2}=1.20 \%\right.$ instead of $2.12 \%$ initially), the roll-off induced by the HAC controller in the outer loop will tend to attenuate the second resonant mode amplitude afterwards.

Fig. 16 shows experimental impulse responses for the uncontrolled and controlled microactuator, proving that the proposed PPF controller is capable of successfully attenuating a part of the vibrations of the structure.

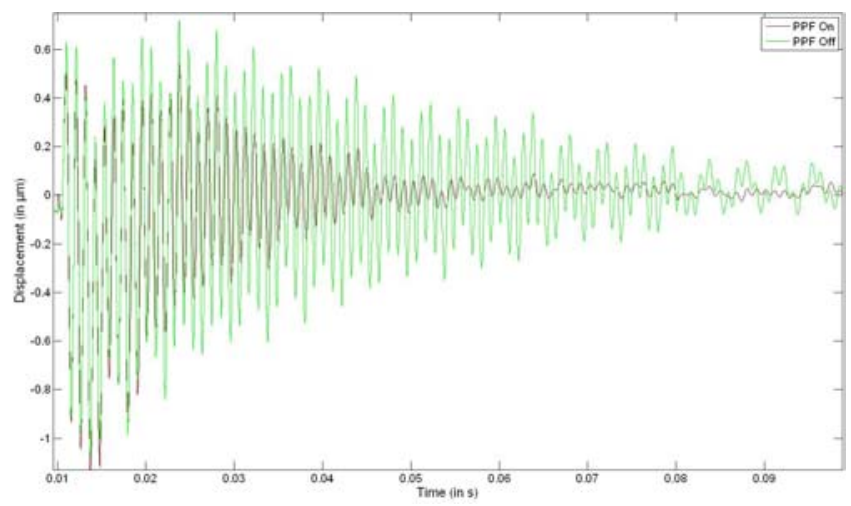

Fig. 16. Impulse responses for the uncompensated and PPF pre-compensated microactuator 


\section{B. HAC regulator synthesis using frequency shaping}

PPF schemes are very effective to improve modal damping. But, away from the resonances, active damping is completely ineffective, because it leaves the close-loop poles of the system almost unchanged [12] [31]. To cause substantial pole displacement, we have chosen to use a second regulator named HAC, whose design methodology is explained as follows :

- To increase the gain at low frequency, the HAC compensator includes an integrator $1 / s$, allowing to eliminate the static error and to reject disturbance. In high-frequency, it provides sensor noise rejection and stability robustness too.

- A pre-multiplicative term has been added to the previous integrator to increase the bandwidth $\omega_{B . W}$. of the external loop transfer

$$
L(s)=\frac{H_{L}(s) G(s)}{1-H_{l}(s) G(s)}
$$

up to $\omega_{B . W}$. $\geq 200 \mathrm{rad} / \mathrm{s}$.

- A lag compensator has been finally tuned to reduce the gain of the loop transfer at high frequency. A $(\pi / 9)$ phase lag centered on the second flexible mode resonance $\omega_{r 2}=$ $5875 \mathrm{rad} / \mathrm{s}$ is added. It increases the gain margin between the second resonant peak amplitude and the $0 \mathrm{~dB}$ value, without deteriorating too much the bandwidth $\omega_{B . W .}$.

The resulting regulator

$$
H_{L}(s)=2389\left(\frac{1}{s}\right)\left(\frac{1+0.00012 s}{1+0.00024 s}\right)
$$

models the $L(s)$ loop transfer as seen on Fig.17, providing interesting gain and phase margins $\left(M_{g}=16.60 \mathrm{~dB}\right.$ and $M_{\varphi}=78.62 \mathrm{deg}$ at $\omega_{P}=217.56 \mathrm{rad} / \mathrm{s}$ respectively).

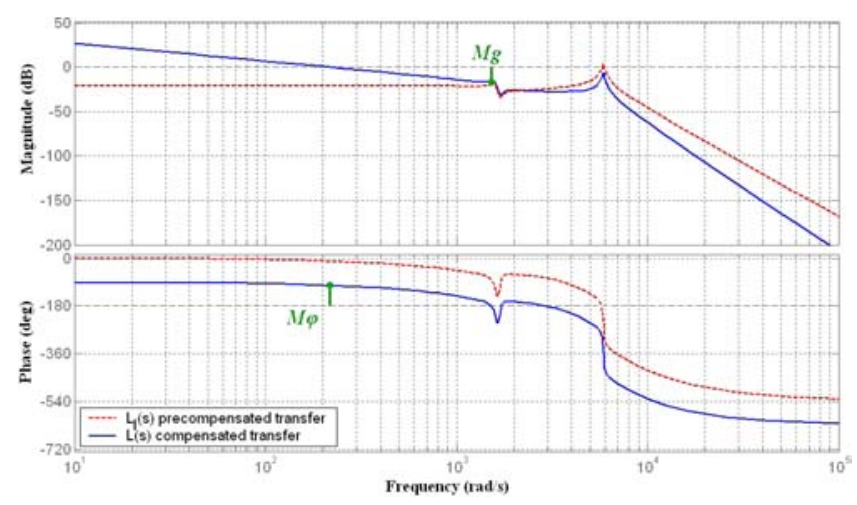

Fig. 17. Comparison between the $L_{l}(s)=\frac{G(s)}{1-H_{l}(s) G(s)}$ pre-compensated loop transfer (considering only LAC regulator influence) and the $L(s)$ totally compensated loop transfer (considering the LAC and HAC regulators influence).

\section{Experimental results}

Reference steps of various amplitudes were experimentally applied on the device Fig.18 to test its robustness. The tracking performances are validated with a settling time of $t_{5 \%}=13 \mathrm{~ms}$ which is about three times more rapid than the open-loop $t_{5 \%}$.

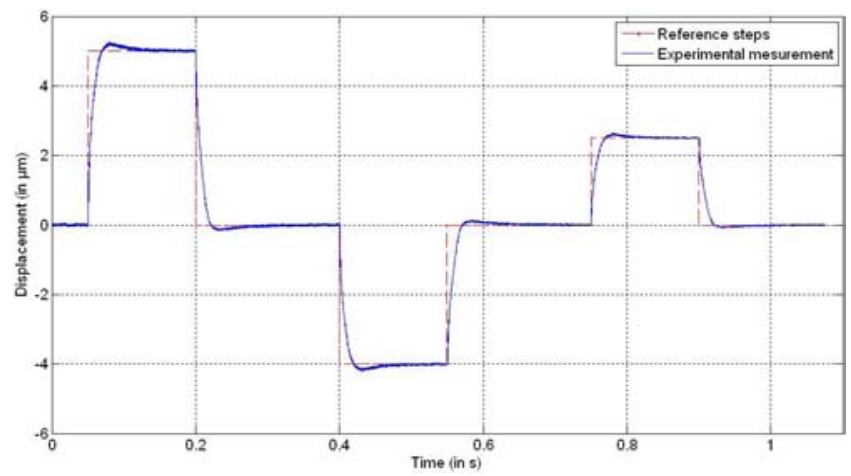

Fig. 18. Experimental step responses of the HAC/LAC compensated microactuator.

\section{RST ROBUST CONTROL OF THE MICROACTUATOR}

In this section, a controller synthesis, based on a robust poles placement, is presented. Then, it is implemented and tested on the device to allow comparisons with the previous control HAC/LAC strategy.

The synthesized controller is based on the robust poles placement architecture in continuous domain [32]. At the end, the design methodology provides a polynomial regulator under the RST form (see Fig.19), where $A$ and $B$ polynomials are defined by the input/output transfer $G$, as follows

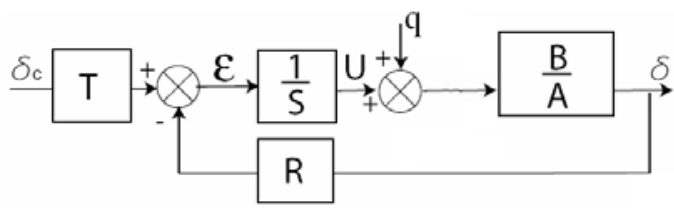

Fig. 19. Equivalent polynomials RST controller.

$$
\frac{\delta(s)}{U(s)}=G(s)=\frac{B(s)}{A(s)}
$$

The close-loop transfer between output deflection $\delta$, input reference $\delta_{c}$, and disturbance $\mathrm{q}(\mathrm{s})=\frac{\delta_{P e r t} .(\mathrm{s})}{\alpha_{O} D(\mathrm{~s})}$ results in

$$
\delta(s)=\frac{B(s) T(s)}{D_{s}(s)} \delta_{c}(s)+\frac{B(s) S(s)}{D_{s}(s)} q(s)
$$

In (18), the specified assignment polynomial $D_{s}$ is expressed in function of the unknown polynomials $R$ and $S$ under the Bezout identity to solve:

$$
D_{s}(s)=A(s) S(s)+B(s) R(s)
$$

The assigned close-loop poles of $D_{s}(s)$ are chosen with the robustness, performance and stability objectives in mind. Amongst the different existing approaches [33], the proposed one here is inspired by [32]. The polynomial coefficients are calculated looking at the roots of the polynomial with some control and filtering horizon parameters.

Although control horizon will tend to be as small as possible, limitations come from the command energy amplitude on the one hand, and robustness performances on the other hand. Robustness is generally improved when 
increasing the filtering horizon to the detriment of the regulation performances. In our study, simulations tests have been carried out to get about the same settling time for pursuit transfer as for the previous HAC/LAC compensated system. To avoid oscillations in time response of the system, the specified damping is taken equal to 1 . Corresponding stability margins are important $\left(M_{g}=23.22 d B\right.$ and $M_{\varphi}=82.30 \mathrm{deg}$ at $\left.\omega_{\varphi}=25 \mathrm{rad} / \mathrm{s}\right)$.

Step references of $5 \mu \mathrm{m}$ were experimentally tested on the device Fig.20. The tracking performances are validated with an experimental settling time of $t_{5 \%}=11 \mathrm{~ms}$.

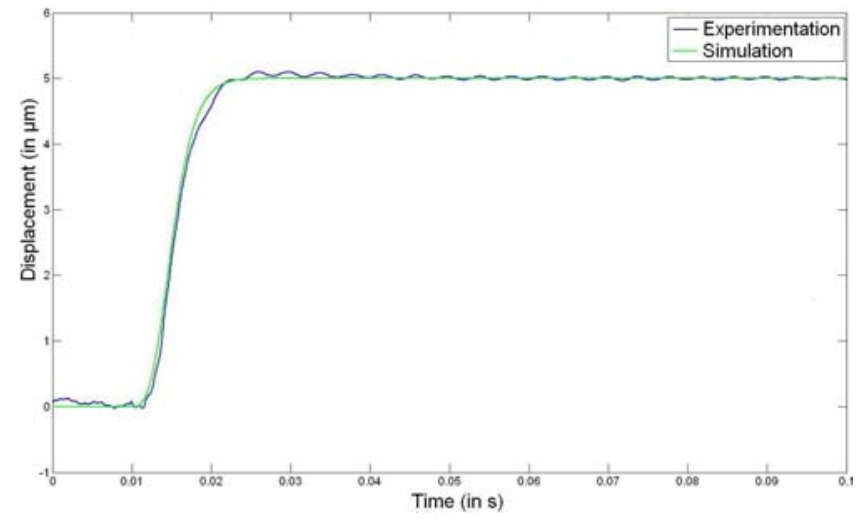

Fig. 20. Experimental and simulated step responses of the RST compensated microactuator. (Step references of $5 \mu \mathrm{m}$ starting at $t=0.01 \mathrm{~s}$.)

\section{DISCUSSIONS AND CONCLUSIONS}

Robust tracking control of a nonlinear piezoelectric system is presented in this paper. The model used for the synthesis of the robust controllers takes into account gripping forces, creep and hysteresis phenomena as external disturbances. The latter effect is viewed here as a quadrilateral approximated model for the static hysteresis part. This static part is subjected to uncertainty and to a varying static gain. The dynamic part of the frequency model is modeled using a reduced accurate model, thanks to the optimization made previously on our device [3]. Such a modeling choice leads to a simple model which is very suitable for linear controller design.

Taking advantages of the interlacing pole-zero pattern of the device, a mixed Low Authority Control and High Authority Control strategy has been demonstrated to be effective in damping the first dominant flexible mode and in robustly achieving motion tracking.

The experimental performances reached by this approach are as good as the ones obtained by one advanced robust control technique (robust pole placement), making it suitable for micromanipulation tasks.

Indeed, the robustness margins $M_{g}$ and $M_{\varphi}$ are almost equivalent for the RST-based and HAC/LAC controllers, as shown in Table.II. All the step responses are well reproductive and show that tracking performances are correctly achieved with similar settling times for the two control techniques.
TABLE II

ROBUSTNESS MARGINS COMPARISON

\begin{tabular}{c|cc}
\hline Controller & $M_{g}(d B)$ & $M_{\varphi}($ deg $)$ \\
\hline \hline HAC/LAC & 16.60 & 78.62 \\
\hline RST & 23.22 & 82.30 \\
\hline
\end{tabular}

Thus, it emphasizes the fact that, in our specific case of frequency-optimized device, integral action on the PPF augmented system (HAC/LAC strategy) gives about the same interesting performances as those obtained with RST-based robust poles placement technique.

Moreover, tuning of the PPF is easily done using root locus technique. The way we choose to model the hysteresis curve with quadrilateral approximation enables to easily guarantee stability towards hysteresis parametric uncertainties.

An other major interest of the HAC/LAC approach is the low regulator order obtained (of degree 4), in comparison with the standard $R S T$. Indeed, robust poles placement techniques imposes that the degrees of the $R S T$ compensator fractions are at least twice the degree of the system to be controlled. In our case, the order of the identified transfer $\alpha_{0} D(s)$ (limited to the first two resonant modes) is already equal to 4 , without taking into account the anti-aliasing filter. It inevitably leads to higher order controller afterwards.

Our HAC/LAC approach is quite original and not a widespread technique in the literature dealing with the control of flexible microactuators. In comparison with classic robust control approaches, our approach is competitive in terms of robustness and performances as well. The HAC/LAC controller can be easily tuned using the root locus technique and leads to low order regulator at the end.

Thus, a natural perspective with our prototype deals with the possible integration of this controller in a system-on-chip.

\section{REFERENCES}

[1] Agnus J., Nectoux P., Chaillet N., "Overview of microgrippers and micromanipulation station based on a MMOC microgripper", Proceedings of the IEEE International Symposium on Computational Intelligence in Robotics and Automation, CIRA, Espoo, Finland, 2005, pp. 117-123.

[2] Breguet J.M., and al., "Monolithic piezoceramic flexible structure for micromanipulation", 9th International Precision Engineering Seminar and 4th International Conference on Ultraprecision in Manufacturing Engineering, pp. 397-400, Braunschweig, Germany, 1997.

[3] Grossard M., Rotinat-Libersa C., Chaillet N., Boukallel M., "Mechanical and control-oriented design of a monolithic piezoelectric microgripper using a new topological optimisation method", IEEE/ASME Transactions on Mechatronics, vol. 14, pp.32-45, 2009.

[4] Grossard M., Rotinat-Libersa C., Chaillet N., "Gramian-based optimal design of a dynamic stroke amplifier compliant micro-mechanism", IEEE/RSJ International Conference on Robots and Systems, San Diego, USA, 2007.

[5] P. Bernardoni, P. Bidaud, C. Bidard, F. Gosselin, "A new compliant mechanism design methodology based on flexible building blocks", in Proc. SPIE, Smart Structures and Materials: Modeling, Signal Processing, and Control, San Diego, CA, 2004, vol. 5383, pp. 244-254.

[6] Grossard M., Rotinat-Libersa C., Chaillet N., "Redesign of the MMOC microgripper piezoactuator using a new topological method", IEEE/ASME International Conference on Advanced Intelligent Mechatronics, Zürich, Switzerland, 2007. 
[7] J. Zhong, S. Seelecke, R. C. Smith and C. Büskens, "Optimal control of piezoceramic actuators", in Proc. SPIE, Smart Structures and Materials Modeling, Signal Processing, and Control, San Diego, CA, 2003, vol. 5049, pp. 264-274.

[8] J. A. Main, E. Garcia and D. V. Newton, "Precision position control of piezoelectric actuators using charge feedback", Journal of guidance, Control and Dynamics, 18(5):1068-1073, 1995.

[9] P. Ge and M. Jouaneh, "Generalised preisach model for hysteresis nonlinearity of piezoelectric actuators", Precision Engineering, 20, 99111, 1997.

[10] Y. Gao and S. Tse, "Modelling of piezoelectric actuator based nanopositioning system under sinusoidal excitation using multi-olynomial regression", Journal of Physics: Conference Series 13, pp.98-101, 2005.

[11] Robinson R. S., "Interactive computer correction of piezoelectric creep in scanning tunneling microscopy images", J. Comput.-Assist. Microsc., pp. 53-58, 1996

[12] Preumont A., "Vibration control of active structures: an introduction 2nd edition", Kluwer academic publishers, 2002.

[13] Gevarter W. B., "Basic relations for control of flexible vehicles", AIAA Journal, vol. 8, pp. 666-672, 1970

[14] Martin G. D., "On the control of flexible mechanical systems", $\mathrm{PhD}$ Dissertation, Stanford University, USA, 1978.

[15] Aphale S.S., Fleming A.J., Moheimani S. O. R., "Integral resonant control of collocated smart structures", Smart Materials and Structures, vol.16, pp. 439-446, 2007.

[16] PI Piezo Ceramic Technology, 2005. Available: http://www.piceramic.com/.

[17] Croft D., Shed G., Desavia S., "Creep, hysteresis and vibration compensation for piezoactuators: atomic force microscopy applications", Journal of Dynamic Systems, Measurements and Control, Vol. 123, Issue 1, pp.35-43, March 2001.

[18] Dimmler M., Holmberg U., Longchamp R. L., "Hysteresis compensation of piezo-actuators", in Proc. 5th European Control Conf., 1999, CDROM paper f0700.

[19] Pons J. L., "Emerging actuator technologies: a micromechatronic approach", ISBN 0-470-09197-5, Wiley, 2005.

[20] Klinkel S., "A phenomenological constitutive model for ferroelastic and ferroelectric hysteresis effects in ferroelectric ceramics", International Journal of Solids and Structures, Volume 43, Issues 22-23, pp. $7197-$ 7222, 2006.

[21] Qing-Ming W., Xiao-Hong D., Baomin X. and Cross L.E., "Electromechanical coupling and output efficiency of piezoelectric bending actuators", IEEE Transactions on Ultrasonics, Ferroelectrics and Frequency Control, Vol. 46, Issue 3, pp. 638-646, 1999.

[22] Rakotondrabe M., Haddab Y., Lutz P., "Quadrilateral modelling and robust control of a non-linear piezoelectric cantilever", IEEE Transactions on Control Systems Technology, Vol. 17 (3), pp. 528-539, 2009.

[23] Rakotondrabe M., Haddab Y., Lutz P., "Non-linear modeling and estimation of force in a piezoelectric cantilever", IEEE/ASME Internationale Conference on Advanced Intelligent Mechatronics, 4-7 September, 2007.

[24] Devasia S., Eleftheriou E. and Moheimani S. O. R.,"A Survey of Control Issues in Nanopositioning", IEEE Transactions on Control Systems Technology, Vol. 15, pp. 802-823, 2007.

[25] Madill D. R., Wang D., "Modeling and L2-stability of a shape memory alloy position control system", IEEE Transactions on Control Systems Technology, vol. 6, pp. 473-481, July 1998.

[26] Pozzi M., King T., "Dynamic characteristics of piezoelectric multilayer stack actuators",in Proc. 2nd Int. Recent Advances Mechatron. Conf., pp. 461-466, 1999.

[27] Coleman B. D. and Hodgdon M. L., "A constitutive relation for ratedependent hysteresis in ferromagnetically soft materials", Int. J. Eng. Sci., pp. 897-919, 1986.

[28] Banningand R., Koning W. L., Adriaens J. M. T. A., and Koops K. R., "Statespace analysis and identification for a class of hysteretic systems". Automatica, pp.1883-1892, 2001.

[29] Fanson J. L., Caughey T. K., "Positive position feedback-control for large space structures", AIAA Journal, vol. 28, pp. 717-724, 1990.

[30] Friswell M., Inman D.J., "The relationship between positive position feedback and output feedback controllers", Smart. Mater. Struct., vol. 8 , pp. 285Ü291, 1999.

[31] Pang C. K., Guo G., Chen M. and Lee T. H., "Nanoposition sensing and control in HDD dual-stage servo systems", IEEE International Confcrence on Control Applications Taipei, pp.551-556, Taiwan, September 2-4, 2004.

[32] De Larminat P., Puren S., "Robust poles placement design via LTR approach", Proceedings of the 14th IFAC World Congress, Beijing, July 1999.
[33] Astrom K., Wittenmark B., Computer controlled systems, theory and design, Prentice Hall, 1984. 